\title{
Enhancement of gefitinib-induced growth inhibition by Marsdenia tenacissima extract in non-small cell lung cancer cells expressing wild or mutant EGFR
}

Shu-Yan Han ${ }^{1}$, Hui-Rong Ding ${ }^{2}$, Wei Zhao ${ }^{3}$, Fei Teng ${ }^{1}$ and Ping-Ping Li ${ }^{1 *}$

\begin{abstract}
Background: Non-small cell lung cancer (NSCLC) expressed high levels of epidermal growth factor receptor (EGFR). Gefitinib (Iressa) has demonstrated clinical efficacy in NSCLC patients harboring EGFR mutations or refractory to chemotherapy. However, most of NSCLC patients are with wild type EGFR, and showed limited response to gefitinib. Therefore, to develop new effective therapeutic interventions for NSCLC is still required. Our previous study showed Marsdenia tenacissima extract (MTE) restored gefitinib efficacy in the resistant NSCLC cells, but whether MTE acts in the gefitinib-sensitive NSCLC cells is the same as it in the resistant one is unknown.
\end{abstract}

Methods: Dose response curves for gefitinib and MTE were generated for two sensitive NSCLC cell lines with mutant or wild type EGFR status. Three different sequential combinations of MTE and gefitinib on cell growth were evaluated using $\mathrm{I}_{50}$ and Combination Index approaches. The flow cytometric method was used to detect cell apoptosis and cell cycle profile. The impact of MTE combined with gefitinib on cell molecular network response was studied by Western blotting.

Results: Unlike in the resistant NSCLC cells, our results revealed that low cytotoxic dose of MTE $(8 \mathrm{mg} / \mathrm{ml})$ combined gefitinib with three different schedules synergistically or additively enhanced the growth inhibition of gefitinib. Among which, MTE $\rightarrow$ MTE + gefitinib treatment was the most effective one. MTE markedly prompted cell cycle arrest and apoptosis caused by gefitinib both in EGFR mutant (HCC827) and wild type of NSCLC cells (H292). The Western blotting results showed that MTE $\rightarrow$ MTE + gefitinib treatment further enhanced the suppression of gefitinib on cell growth and apoptosis pathway such as ERK1/2 and PI3K/Akt/mTOR. This combination also blocked the activation of EGFR and c-Met which have cross-talk with each other. Unlike in gefitinib-resistant NSCLC cells, MTE alone also demonstrated certain unexpected modulation on EGFR related cell signal pathways in the sensitive cells.

Conclusion: Our results suggest that MTE is a promising herbal medicine to improve gefitinib efficacy in NSCLC regardless of EGFR status. However, why MTE acted differently between gefitinib-sensitive and -resistant NSCLC cells needs a further research.

Keywords: Marsdenia tenacissima extract (MTE), Gefitinib, Non-small cell lung cancer (NSCLC), Combination, EGFR related pathway

\footnotetext{
* Correspondence: Ippma123@sina.com

'Key Laboratory of Carcinogenesis and Translational Research (Ministry of Education), Department of Integration of Chinese and Western Medicine, Peking University Cancer Hospital \& Institute, No. 52 Fucheng Road, 100142 Haidian District, Beijing, P.R. China

Full list of author information is available at the end of the article
} 


\section{Background}

Lung cancer is the leading cause of cancer death worldwide. The high mortality of lung cancer is related to the fact that most patients present with metastatic disease for which there is no curative therapy. Non-small cell lung cancer (NSCLC) accounts for 75\% - 80\% of all lung cancers [1]. Epidermal growth factor receptor (EGFR) is a family member of EGF-related tyrosine kinase receptors, and expressed at high levels in many cancer cell types, including NSCLC [2]. This leads to inappropriate activation of the downstream signalling cascade, eventually leading to uncontrolled cell proliferation [3]. Studies showed EGFR is overexpressed in up to $80 \%$ of NSCLC and become a promising target for anti-cancer therapy $[4,5]$.

An orally active tyrosine kinase inhibitor (TKI), gefitinib (ZD1839, Iressa), competes with ATP for the binding sites at tyrosine kinase domain, thereby dampening the phosphorylation and activation of EGFR so as to the downstream signaling network [6,7]. Gefitinib has been shown to significantly improve progression-free survival and used extensively for the first-line therapy in advanced NSCLC patients harboring EGFR mutations [8,9]. Exon 19 deletion mutations and L858 mutation in exon 21 of EGFR increase gefitinib sensitivity in NSCLC [10,11]. However, only $10 \%$ - 20\% NSCLC patients with wild type of EGFR responded to gefitinib [12,13]. Moreover, clinical study revealed that NSCLC patients with EGFR mutations have a significantly longer survival than those with wildtype EGFR when treated with EGFR TKIs [14]. Despite experiencing dramatic clinical responses, patients who initially respond to gefitinib eventually develop progressive disease or incomplete cross-resistance to the currently available EGFR-TKIs like erlotinib $[15,16]$. Therefore, how to improve gefitinib efficacy and let more NSCLC patietns gain benefit from TKI therapy is still the goal of physicians and researchers.

Marsdenia tenacissima (Roxb.) Wight et Arn., which mainly produced in Yunnan province of China, has long been used as a remedy to treat cancer in China [17]. More than $40 \mathrm{C}-21$ steroidal glycosides have been isolated from the stem of M. tenacissima [18]. C-21 steroids have previously been shown to be cytotoxic in cancer cell lines [19] suggesting that $\mathrm{C}-21$ steroids are responsible for the anticancer activities of $M$. tenacissima. Thirteen of the C-21 steroid compounds were identified in MTE by HPLC-MS/ MS analysis [20]. Xiao-Ai-Ping injection, a water extract of $M$. tenacissima, has been proposed as a potential agent in the management of tumors, which is clinically effective in treatment of NSCLC when combined with chemotherapy [21-23]. Commonly, the treatment dosage of Marsdenia tenacissima extract (MTE) for cancer patients is between $20 \mathrm{ml}$ to $100 \mathrm{ml}$ (equals to $20 \sim 100$ g crude drug) per day according to the manufacture's instruction. According to our previous study, MTE restores gefitinib sensitivity in the resistant NSCLC cells and the mechanisms may be partially due to the down-regulation of PI3K/Akt/ mTOR and ERK1/2 and inhibition of c-Met phosphorylation [20]. However, whether MTE could enhance gefitinib efficacy in the sensitive NSCLC cells is unknown, and whether the mode of action of MTE show difference or just the same in gefitinib-sensitive and -resistant NSCLC cells.

Therefore, the present study was to evaluate the regimen of MTE sequential combination with gefitinib against gefitinib-sensitive NSCLC cells, HCC827 (EGFR mutant) and H292 (wild type EGFR), and to seek the possible mechanisms may involve.

\section{Methods \\ Cell culture}

Human NSCLC cell lines HCC827 (epithelial adenocarcinoma) and H292 (alveolar epithelial carcinoma) were purchased from American Type Culture Collection (Manassas, Virginia, USA). H292 cells contains wildtype EGFR, whereas HCC827 bearing EGFR exon 19 deletion [24]. Cells were maintained in RPMI-1640 (Gibco) supplemented with $10 \%$ heat-inactivated fetal bovine serum (FBS) (Gibco), 100 units $/ \mathrm{ml}$ penicillin, and $100 \mu \mathrm{g} / \mathrm{ml}$ streptomycin at $37^{\circ} \mathrm{C}$ and $5 \% \mathrm{CO}_{2}$.

\section{Drug treatment}

Gefitinib was purchased from AstraZeneca (Cheshire, UK). Stock solution was prepared in dimethyl sulfoxide (DMSO) at $20 \mathrm{mM}$ and stored at $-20^{\circ} \mathrm{C}$. MTE (M. tenacissima extract, trade name: Xiao-Ai-Ping injection) (1 g crude/ml) was produced by SanHome Pharmaceutical Co., Ltd (NanJing, China). When the cells were treated with MTE or gefitinib, the MTE solvent or $0.5 \%$ DMSO was used as the negative control accordingly.

\section{Antibodies and reagents}

The specific antibody of EGFR, p-EGFR (Tyr1068), pPI3K, Akt, mTOR, p-mTOR (Ser2448), Met, p-Met (Y1234/1235), ERK1/2, p-ERK1/2, $\beta$-actin and $\beta$-tubulin were purchased from Cell Signaling Technology (Beverly, MA). PI3K and p-Akt (Ser473) were obtained from ABcam (Cambridge, UK). The enhanced chemiluminescence (ECL) system was from Millipore (Millipore, MA, USA). Epidermal growth factor (EGF) was purchased from Biosource International Inc. (Camarillo, CA) and dissolved in phosphate-buffered saline (PBS).

\section{Cell viability assay}

The cell viability was measured by MTT assay. In brief, cells were plated in 96-well culture plates at the density of $1 \sim 1.5 \times 10^{4}$ per well in complete medium. After $24 \mathrm{~h}$ incubation, cells were treated with gefitinib $(0.001 \sim 50 \mu \mathrm{M})$ or MTE $(0.5 \sim 500 \mathrm{mg} / \mathrm{ml})$ for $72 \mathrm{~h}$. The optical density at $570 \mathrm{~nm}$ was measured and the $\mathrm{IC}_{50}$ value was calculated 
based on the non-linear regression fit method by Graphpad Prism 4.0 software (San Diego, CA).

\section{Sequential combination effect evaluation}

Cells were plated in 96-well culture plates and incubated for $24 \mathrm{~h}$, then started treatment by following three different sequential combinations described previously $[20,25]$ : (1) first pretreated cells with MTE for $12 \mathrm{~h}$ and then added gefitinib to MTE-containing medium for another $72 \mathrm{~h}$ $(\mathrm{M} \rightarrow \mathrm{M}+\mathrm{G})$; (2) MTE and gefitinib were added concurrently to the medium and incubated for $72 \mathrm{~h}(\mathrm{M}+\mathrm{G})$; (3) first pretreated with gefitinib for $12 \mathrm{~h}$ and then added MTE to gefitinib-containing medium for another $72 \mathrm{~h}$ $(G \rightarrow G+M)$. Each sequential combination of MTE and gefitinib was characterized by a combination index $(\mathrm{CI})$ as described by Chou [26] and calculated with CompuSyn (ComboSyn, Inc., Paramus, NJ, USA). The CI values were interpreted as follows: CI: 0.1-0.3 strong synergism, CI: 0.30.7 synergism, CI: 0.7-0.85 moderate synergism, CI: 0.850.9 slight synergism, CI: 0.9-1.1 nearly additive, and $\mathrm{CI}>1.1$ antagonism.

\section{Cell cycle analysis}

H292 or HCC827 cells were pretreated with MTE for $12 \mathrm{~h}$ and then co-treated with or without gefitinib for another $72 \mathrm{~h}$. Cells were detached with 0.25\% EDTAtrypsin (Gibco), washed with PBS and fixed with icecold $70 \%$ ethanol overnight at $4^{\circ} \mathrm{C}$. Cells were washed with PBS and incubated with RNase A $(100 \mu \mathrm{g} / \mathrm{ml})$ at $37^{\circ} \mathrm{C}$ for $30 \mathrm{~min}$. DNA was labeled with PI $(50 \mu \mathrm{g} / \mathrm{ml})$ and the fluorescence was measured with a FACScalibur flow cytometer (Becton Dickinson). Data collection and analysis of the cell cycle distribution were performed using CellQuest and the Modfit software (Becton Dickinson).

\section{Detection of apoptosis by flow cytometry}

H292 or HCC827 cells were pretreated with MTE for $12 \mathrm{~h}$ and then co-treated with or without gefitinib for another $72 \mathrm{~h}$. Floating and adherent cells were collected and suspended in PBS, labeled with Annexin V and propidiumiodide (PI) following the manufacturer's instruction (Biosea Biotechnology, Beijing, China). Flow cytometry (Bection Dikinson, USA) was used to assess the apoptotic cells. The quantitation of apoptotic cells was calculated by CellQuest software.

\section{Western blot analysis}

After different drug treatment, the cells were stimulated with $10 \mathrm{ng} / \mathrm{ml} \mathrm{EGF}$ for $15 \mathrm{~min}$ before harvesting, washed twice with cold PBS and lysed with RIPA buffer that containing protease and phosphotase inhibitors cocktail (Roche, UK). The supernatants were collected after centrifugation at $12000 \mathrm{rpm}$ for $20 \mathrm{~min}$. The protein was applied to polyacrylamide gel electrophoresis (SDS-
PAGE), transferred to a PVDF membrane, and then detected by the proper primary and secondary antibodies before visualization with a chemiluminescence kit. The intensity of blot signals was quantitated using ImageQuant TL analysis software (General Electric, UK).

\section{Statistical analysis}

All data were expressed as means \pm standard error of the mean (SEM) obtained from at least three independent experiments. Statistical comparisons between experimental and control groups were assessed by using the Student's $t$ test. $P<0.05$ was considered statistically significant.

\section{Results}

\section{Anti-proliferative activity of gefitinib and MTE in NSCLC cells}

We examined the in vitro cell growth inhibition of gefitinib or MTE on NSCLC cells by using MTT assay. After treatment for $72 \mathrm{~h}$, the $\mathrm{IC}_{50}$ value for gefitinib was $0.166 \mu \mathrm{M}$ for H292 cells (EGFR wild type), and $0.015 \mu \mathrm{M}$ for HCC827 cells (EGFR exon 19 deletion) (Figure 1A), indicating they are gefitinib-sensitive cell lines. H292 carrying wild-type EGFR but highly sensitive to gefitinib, suggesting that there exists off-target(s) of gefitinib in addition to the commonly accepted target EGFR, which deserves a further investigation. When exposed to MTE, these two cell lines showed a similar dose-dependent inhibition manner on cell viability regardless of the EGFR status (Figure $1 \mathrm{~B}$ ). The $\mathrm{IC}_{50}$ value of MTE was $46.62 \mathrm{mg} / \mathrm{ml}$ for $\mathrm{H} 292$ cells and $48.78 \mathrm{mg} / \mathrm{ml}$ for HCC827 cells, respectively.

\section{Sequential-combined inhibitory effect of gefitinib and MTE in NSCLC cells}

The effect of sequential combination of gefitinib and MTE was examined in $\mathrm{H} 292$ and $\mathrm{HCC} 827$ cells. In the following experiments, MTE is set at a concentration of $8 \mathrm{mg} / \mathrm{ml}$ for HCC827 and H292 cells (approximately $\mathrm{IC}_{15}$ value). At this concentration, MTE showed only weak inhibition of cell growth with the cell viability approximately $90 \%$. Compared with gefitinib or MTE alone, all cells treated with gefitinib plus MTE exhibited decreased viability (Figure 2A and B). The combination index $(\mathrm{CI})$ values were calculated by CompuSyn software as described in Materials and Methods. Linear correlation coefficients ( $r$ values) for all curves were $>0.95$, indicating a high goodness of fit. As shown in Figure 2C, the $C I$ values in $\mathrm{H} 292$ cells treated by $\mathrm{G}+\mathrm{M}$ and $\mathrm{M} \rightarrow$ $M+G$ were all $<1$, indicating that there was a synergistic interaction between gefitinib and MTE in these two sequential combinations. The $C I$ values in $G \rightarrow G+M$ treated $\mathrm{H} 292$ cells predicted antagonism at the lowest gefitinib concentration but nearly additive to synergistic effects at other high concentrations. In Figure 2D, CI values in HCC827 cells treated by three different 

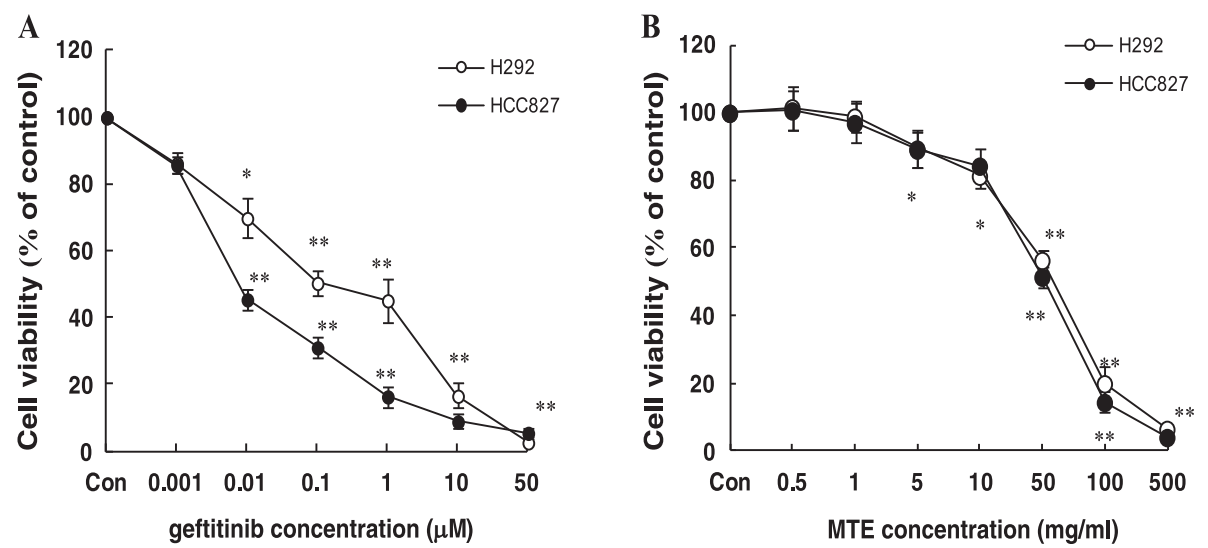

Figure 1 Treatment of non-small cell lung cancer cells with gefitinib or MTE ( $M$. tenacissima extract) reduced their proliferation potential. (A) H292 and HCC827 cells were treated with the indicated concentrations of gefitinib for 72 h. (B) H292 and HCC827 cells were treated with the indicated concentrations of MTE for $72 \mathrm{~h}$. Cells viability was determined by using the MTT assay as described in the Methods section. The data are expressed in terms of percent of control cells as the means \pm SEM. The experiments were repeated at least three times. ${ }^{*} P<0.05,{ }^{* *} P<0.01$ vs. control group.

sequential combinations had same tendency as they were in $\mathrm{H} 292$ cells, but the synergism effects were even more pronounced. Among the three different combinations, the $M \rightarrow M+G$ treatment was potent over the other two sequential combinations both in $\mathrm{H} 292$ and HCC827 cells, indicating this was the preferred combination of gefitinib and MTE.

\section{MTE affected gefitinib on cell cycle distribution}

The effect of MTE combined with gefitinib on cell cycle distribution was examined with flow cytometry. As shown in Figure 3A, MTE treatment caused increase at G2/M phase (from $10.7 \%$ to $13.2 \%$ ) and decrease at G0/ G1 phase (from $71.7 \%$ to $67.0 \%$ ) in $\mathrm{H} 292$ cells when compared with control group. The fraction cells in G2/ $\mathrm{M}$ phase were significantly elevated to $18.6 \%$ and the proportion of G0/G1 phase cells was depressed to $62.5 \%$ by gefitinib alone. Moreover, MTE $\rightarrow \mathrm{MTE}+$ Gef treatment further raised the percentage of G2/M phase cells to $22.9 \%$ and reduced G0/G1 phase cells to $60.7 \%$. The results above suggested that MTE enhanced gefitinib induced G2/M arrest in H292 cells.

Compared with control group, it showed that HCC827 cells treated with MTE induced accumulation in the S phase of cell cycle from $21.5 \%$ to $23.9 \%$ while G2/M phase cells decreased from $16.7 \%$ to $14.1 \%$. $1 \mu \mathrm{M}$ gefitinib treated HCC827 cells were markedly accumulated in the S phase of cell cycle to $26.6 \%$ and the G2/M phase cells were decreased to $11.7 \%$. The percentage of G2/M-phase cells was further decreased to $9.6 \%$ in the MTE $\rightarrow$ MTE + Gef group, and induced a significant cell population in $\mathrm{S}$ phase to $31.0 \%$ (Figure 3B). This finding indicates that cell cycle distribution was blocked significantly in the $\mathrm{S}$ phase when HCC827 cells were treated with MTE combined with gefitinib. Accumulation in the sub-G1 phase of cell cycle were observed both in H292 and HCC827 cells, suggesting the sequential events of cell cycle arrest followed by apoptosis.

\section{MTE enhanced gefitinib-induced apoptosis in NSCLC cells}

To examine the mechanism of inhibitory effect of MTE combined with gefitinib, cell apoptosis was performed by flow cytometry. The final concentration of gefitinib used in the treatments was $1 \mu \mathrm{M}$ because it closes to the serum level observed in patients being treated with gefitinib [27]. Apoptotic cell death was determined in terms of early- or late-stage apoptotic cells, which are shown respectively in the lower right and upper right quadrants of the FACS histograms (Figure 4A). Treatment of H292 and HCC827 cells with gefitinib and MTE alone or in combination induced a substantial fraction of apoptosis. The percentage of total apoptotic cells (including early and late stage) in $\mathrm{H} 292$ cells after different treatment was as follows: $5.8 \%$ (vehicle-treated control), 12.4\% (MTE $8 \mathrm{mg} / \mathrm{ml}$ ), 27.4\% (gefitinib $1 \mu \mathrm{M}$ ), and 66.5\% (MTE $\rightarrow$ MTE + Gef, $M \rightarrow M+G$ ) (Figure 4). Similar tendency of apoptosis was observed in HCC827 cells as shown in Figure 4A and 4B. The combined treatment of MTE and gefitinib further enhanced cell apoptosis that induced by gefitinib in H292 and HCC827 cell lines, and showed significant difference with each drug alone $(P<0.05)$. The results revealed that $\mathrm{M} \rightarrow$ $M+G$ treatment was effective in cell apoptosis induction.

\section{MTE affected EGFR downstream pathways inhibition by gefitinib}

The major pathways activated by EGFR-TK are the RasRaf-MEK-ERK pathway (leading to cell growth), the mTOR pathway (leading to protein synthesis), and the 

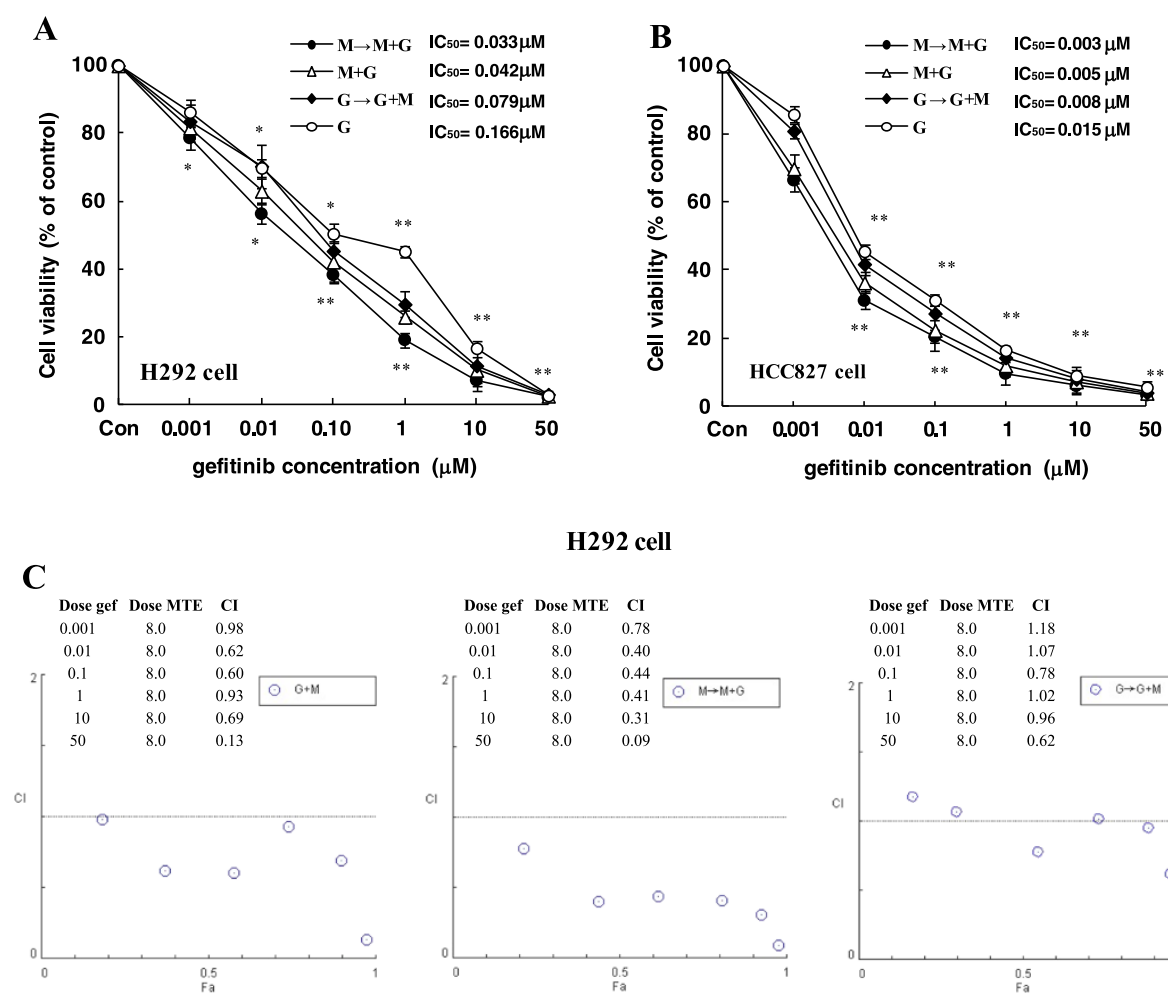

H292 cell
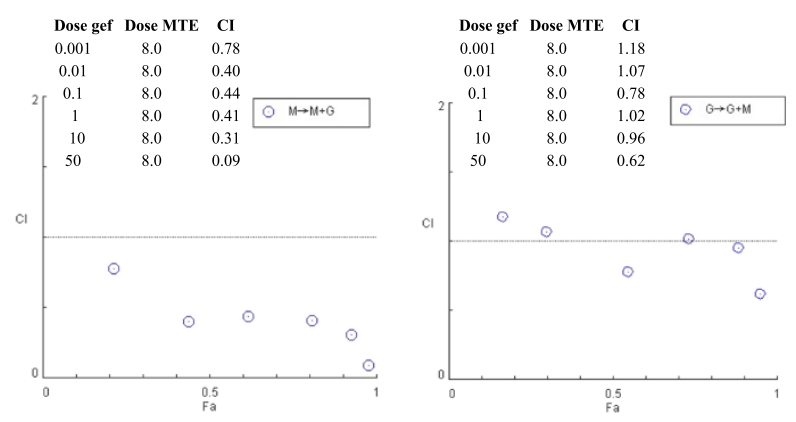

D

$\mathrm{HCC827}$ cell
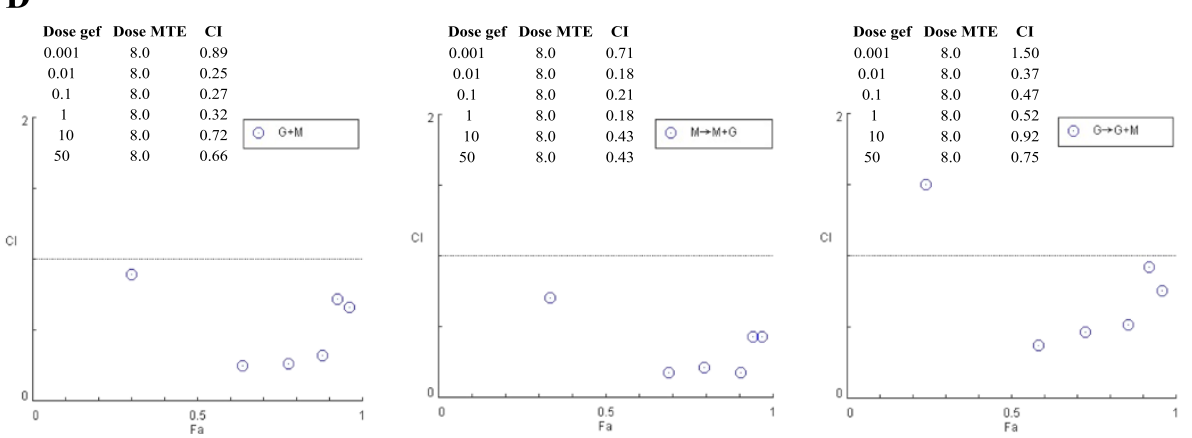

Figure 2 MTE (M. tenacissima extract) enhances gefitinib induced cytotoxicity in gefitinib-sensitive non-small cell lung cancer cell lines. H292 (A) or HCC827 (B) cells were incubated with increasing concentrations of gefitinib and MTE alone or different schedule combinations. Treatment schedule: (1) M $\rightarrow M+G$, MTE pretreated for $12 \mathrm{~h}$, then $M+G$ (MTE + gefitinib) for another $72 \mathrm{~h}$. (2) $M+G$, MTE and gefitinib concomitantly treated for 72 h. (3) $G \rightarrow G+M$, gefitinib pretreated for 12 h, then $G+M$ (gefitinib + MTE) for another 72 h. Growth assays were performed by MTT assay and IC50 values were calculated by Graphpad Prism 5.0 software (San Diego, CA, USA). Cl values for the combinations of gefitinib and MTE in H292 (C) and HCC827 (D) cells were calculated using the Calcusyn software (Cambridge, UK), as described in the Methods section. Each data point represented the mean of 3 to 4 replicates; $r$ values for all curves were $>0.95 .{ }^{*} P<0.05,{ }^{* *} P<0.01$ vs. control group.

PI3K/Akt pathway (leading to cell survival by blocking apoptosis). To investigate the combined effects of MTE and gefitinib on EGFR-dependent signaling pathways, cells were stimulated with $10 \mathrm{ng} / \mathrm{ml}$ EGF for 15 minutes before harvest. We found EGF stimulated the activation of $\mathrm{PI} 3 \mathrm{~K} / \mathrm{Akt} / \mathrm{mTOR}$ and ERK1/2, and their phosphorylation extent is much higher in HCC827 cell (EGFR mutant) than H292 cells (EGFR wide type) (Figure 5). The activation of EGFR downstream pathways was strongly inhibited by gefitinib treatment. Treated with MTE moderately reduced PI3K/Akt/mTOR and ERK1/2 phosphorylation with a similar profile in $\mathrm{H} 292$ and HCC827 cells. Moreover, the inhibition was further enhanced by treatment of $\mathrm{M} \rightarrow \mathrm{M}+\mathrm{G}$ and blocked PI3K/Akt/mTOR and ERK1/2 signaling pathways.

\section{MTE enhanced EGFR and c-Met inhibition by gefitinib}

c-Met and EGFR receptors are widely expressed in cancer cells. The c-Met receptor tyrosine kinase has a central role in the survival of cancer cell and has been identified as a 

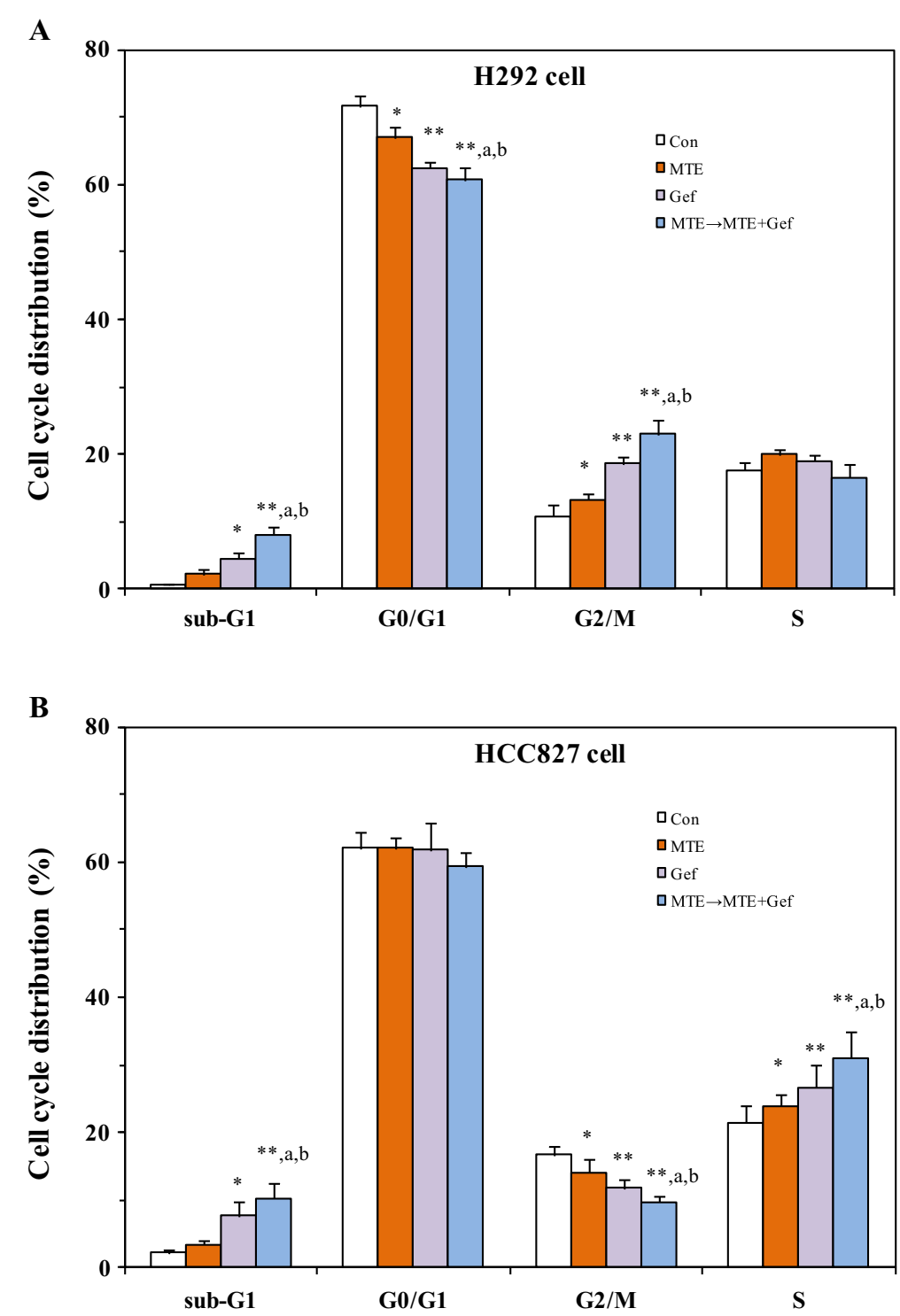

Figure 3 MTE ( $M$. tenacissima extract) enhances gefitinib induced delay in cell cycle in non-small cell lung cancer cell lines H292 (A) and HCC827 (B). Cells were treated with $1 \mu \mathrm{M}$ gefitinib, $8 \mathrm{mg} / \mathrm{ml} \mathrm{MTE}$, and their combination (MTE $\rightarrow$ MTE + Gef, M $\rightarrow$ M + G) in 1\% FBS culture medium for $72 \mathrm{~h}$, then harvested, ethanol fixed and labeled with PI for the analysis of cell cycle by FACS analysis. Each data presented as the means \pm SEM of three experiments. ${ }^{*} P<0.05{ }^{* * *} P<0.01$ vs vehicle control group, ${ }^{a} P<0.05$ vs MTE treated group, ${ }^{b} P<0.05$ vs gefitinib treated group.

novel promising target for NSCLC [28]. It demonstrated that EGFR activation contributes to c-Met tyrosine phosphorylation [29]. Our results showed EGF slightly stimulated the phosphorylation of c-Met and EGFR in H292 and HCC827 cells (Figure 6). However, gefitinib alone or combined with MTE totally blocked the phosphorylation of c-Met and EGFR in these two NSCLC cells. This is in consistent with other research that gefitinib simultaneously inhibited both EGFR and c-Met phosphorylation in gefitinib-sensitive NSCLC cells [30]. Moreover, the activation of EGFR and c-Met was also significantly inhibited by MTE treatment alone as shown in Figure 6. The inhibition on EGFR and c-Met phosphorylation may partly explain the underlying mechanisms of MTE on EGFR-TKI sensitive NSCLC.

\section{Discussion}

The two primary signaling pathways activated by EGFR include the Ras/Raf/MEK/ERK and the PI3K/Akt axes [31]. In the understanding of the nature of NSCLC carcinogenesis, EGFR-TKIs become an innovative approach for NSCLC patients to achieve more effective treatment. Gefitinib exerted anti-tumor effect by inhibiting EGFRdriven signaling activation such as Akt and ERK1/2 in the 


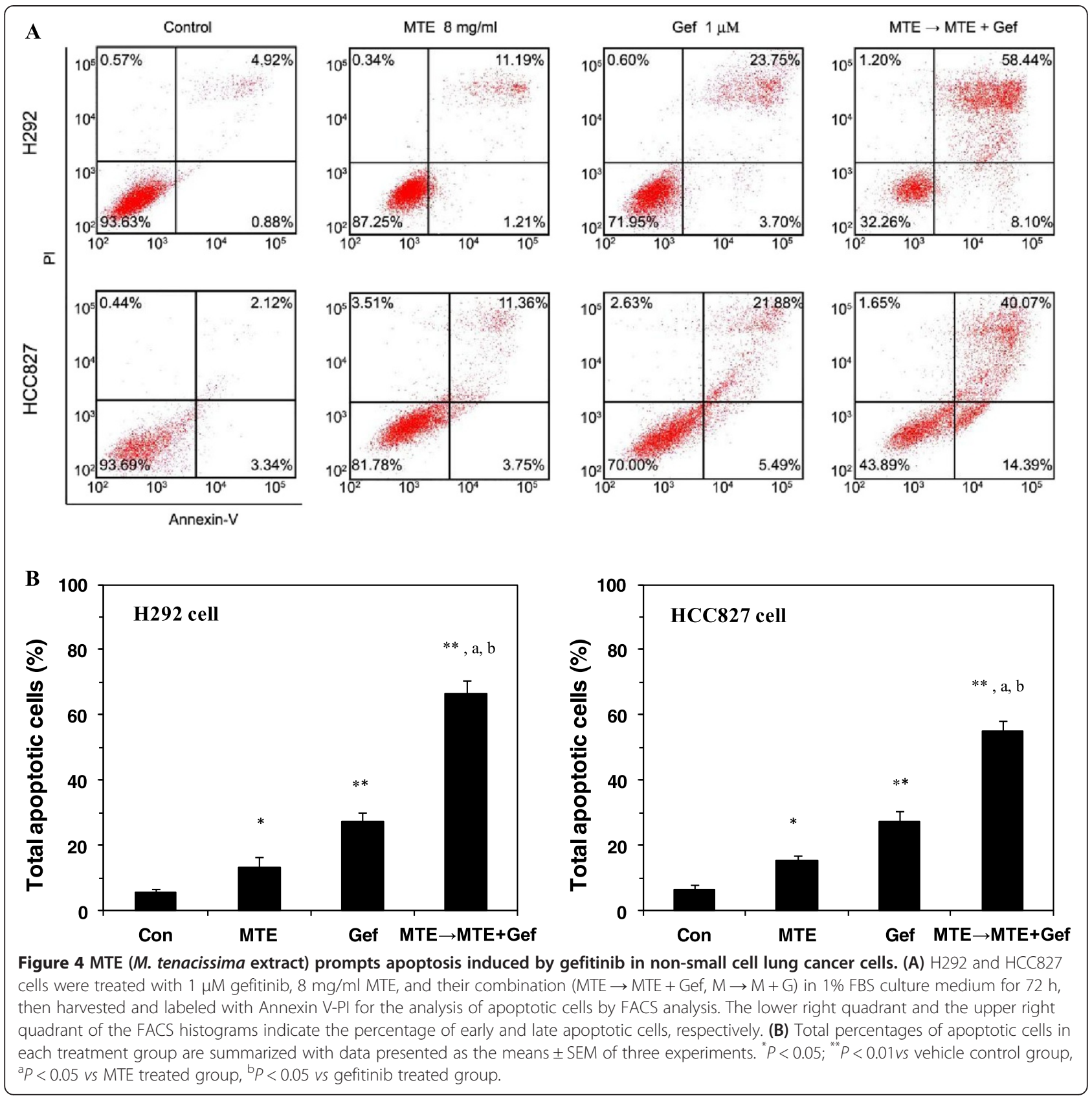

sensitive NSCLC cells [32]. In our research, gefitinib inhibited EGFR and its downstream signaling pathways such as PI3K/Akt/mTOR and ERK in gefitinib-sensitive NSCLC cells, HCC827 and H292, as expected. However, in the resistant NSCLC cells, EGFR is not a survival factor and other factor driven cell survival following activation of downstream signaling effectors was dominant [32].

Nowadays, the use of herbal medicines or compounds from them is becoming increasingly popular, and provides alternative treatment options for cancer patients [33]. It has been demonstrated that curcumin potentiates the antitumor activity of gefitinib in NSCLC cell lines and xenograft mice model [34]. Co-treatment with gefitinib and epigallocatechin gallate (EGCG), a green tea polyphenol, synergistically suppressed the metastatic ability of CAL-27 human oral squamous cell carcinoma cells [35]. We previously reported that M. tenacissima extract (MTE, Xiao-Ai-Ping injection) restored gefitinib sensitivity in the resistant NSCLC cells through synergistic inhibition of PI3K/Akt/mTOR, ERK1/2 activation and c-Met phosphorylation [20]. In the present study, human NSCLC cell line HCC827 and H292 cells were very sensitive to gefitinib with IC50 at $0.015 \mu \mathrm{M}$ and $0.166 \mu \mathrm{M}$, respectively. MTE was also cytotoxic to HCC827 and H292 cells when treated alone. Compared to 


\section{A}
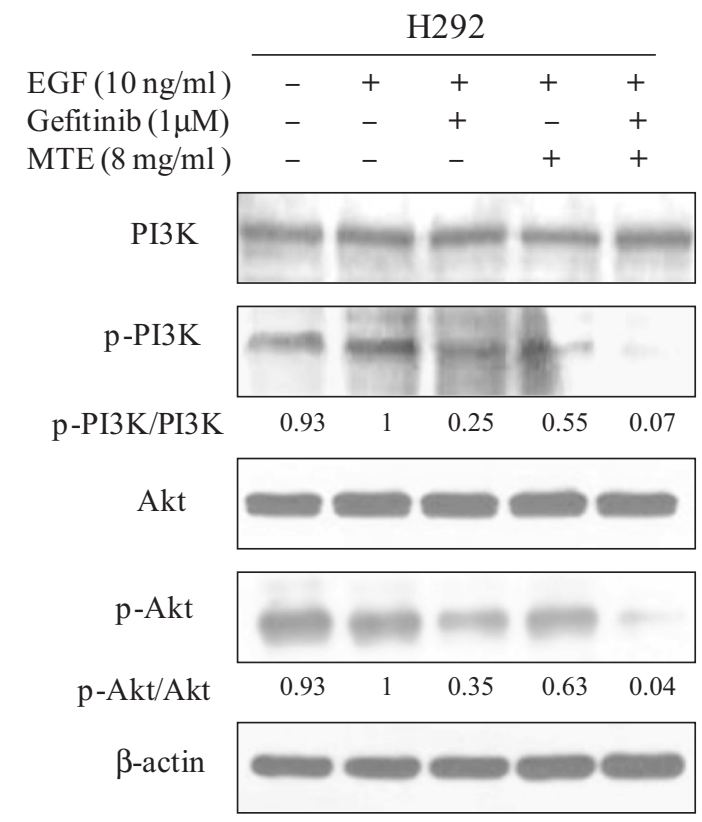

B

$\mathrm{H} 292$

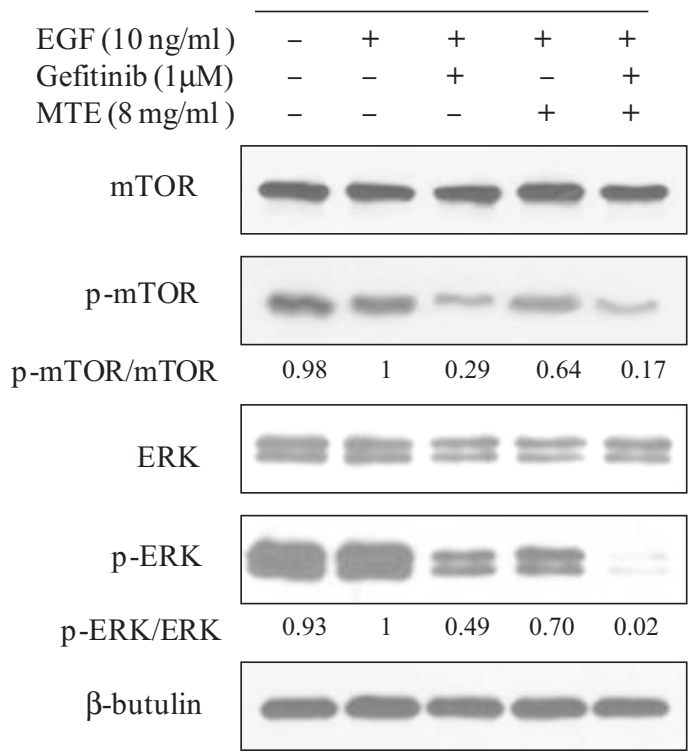

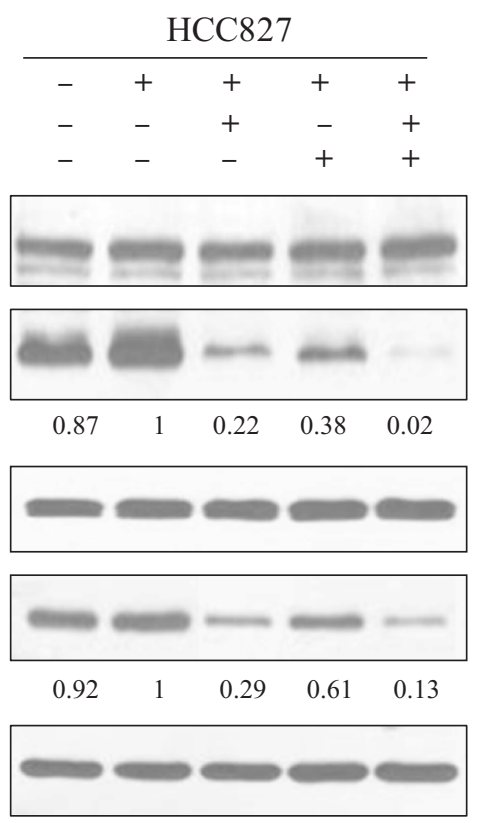
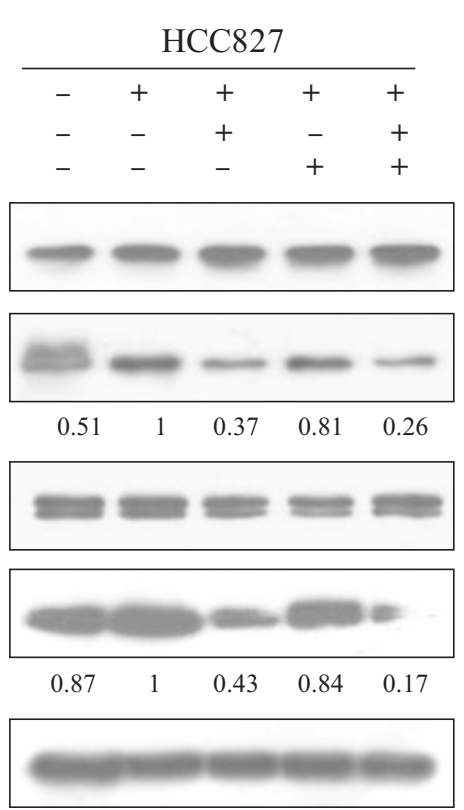

Figure 5 MTE ( $M$. tenacissima extract) enhances the inhibition of gefitinib on PI3K/Akt/mTOR and ERK1/2 signaling cascade in non-small cell lung cancer cells. H292 and HCC827 cells were treated with $1 \mu \mathrm{M}$ gefitinib, $8 \mathrm{mg} / \mathrm{ml}$ MTE, and their combination (MTE $\rightarrow$ MTE + Gef, $\mathrm{M} \rightarrow \mathrm{M}+\mathrm{G}$ ) in $1 \%$ FBS culture medium for $6 \mathrm{~h}$. Cells were stimulates with $10 \mathrm{ng} / \mathrm{ml}$ EGF for $15 \mathrm{~min}$ before harvest. Cells were lysed and cellular extracts (20 $\mathrm{\mu g}$ protein) were separated on SDS-PAGE gel and transferred to PVDF membranes. (A) Membranes were probed with PI3K, phospho-PI3K, Akt and phospho-Akt; (B) Membranes were probed with mTOR, phospho-mTOR, ERK1/2 and phospho-ERK1/2. Blots are representatives of three independent experiments. Relative density of proteins were calculated and normalized based on $\beta$-tubulin or $\beta$-actin.

the antitumor effect of each reagent alone, the combined gefitinib and MTE treatment synergistically or additively suppressing cell viability regardless of the sequential difference. Among which, the MTE $\rightarrow$ MTE +
Gef treatment showed more potent on decreasing cell growth than the other combinations, concurrent administration and Gef $\rightarrow$ Gef + MTE. Furthermore, the enhanced cell proliferation inhibition by MTE $\rightarrow$ MTE + 


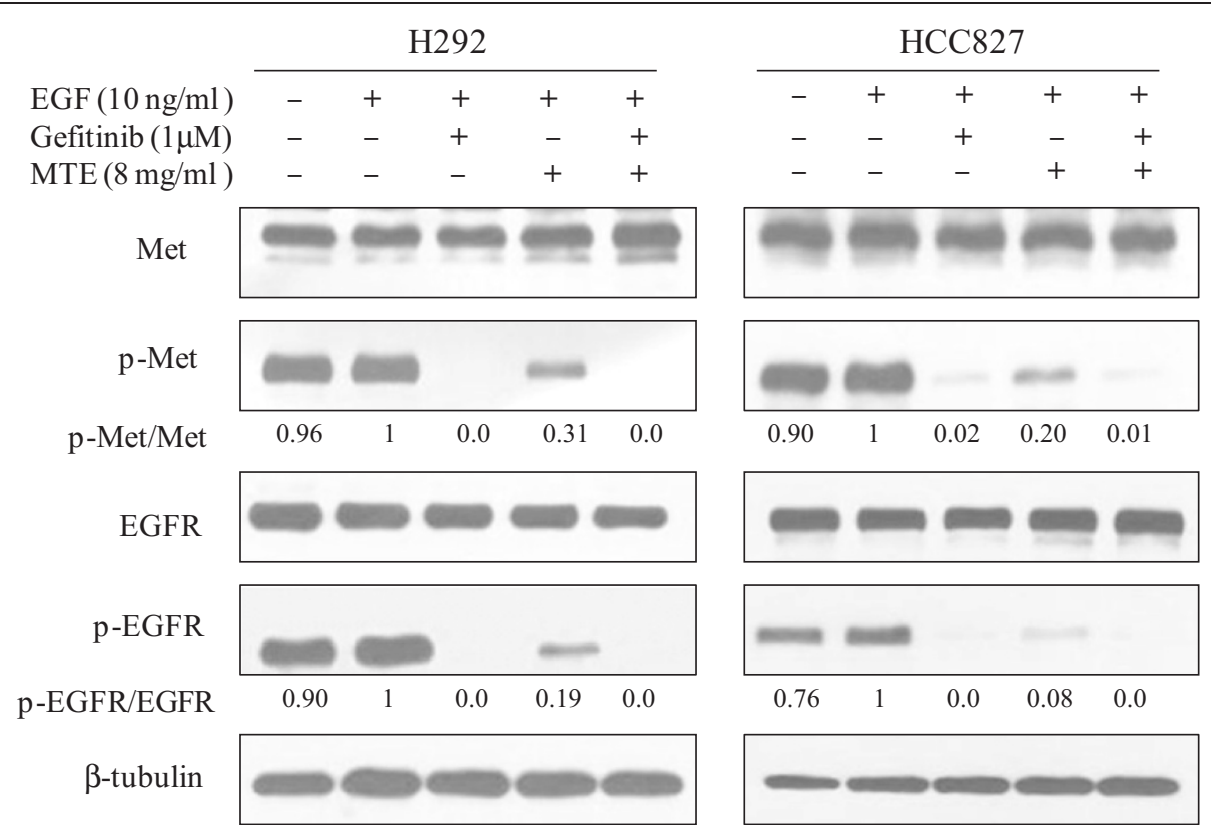

Figure 6 MTE (M. tenacissima extract) combined with gefitinib reduces EGFR and Met crosstalk in non-small cell lung cancer cells. H292 and HCC827 cells were treated with $1 \mu \mathrm{M}$ gefitinib, $8 \mathrm{mg} / \mathrm{ml} \mathrm{MTE}$, and their combination (MTE $\rightarrow$ MTE + Gef) in $1 \%$ FBS culture medium for $6 \mathrm{~h}$. Cells were stimulates with $10 \mathrm{ng} / \mathrm{ml}$ EGF for $15 \mathrm{~min}$ before harvest. Cells were lysed and cellular extracts (20 $\mu \mathrm{g}$ protein) were separated on SDS-PAGE gel and transferred to PVDF membranes. Membranes were sequentially probed with Met, phospho-Met, EGFR and phospho-EGFR. $\beta$-tubulin was served as a loading control. Blots are representatives of three independent experiments.

gefitinib sequential combination was over each agent alone. This is in accordance with our previous result that MTE $\rightarrow$ MTE + Gef had potential effect against gefitinib-resistant NSCLC cell lines [20]. However, it was very interesting that the Gef $\rightarrow$ Gef + MTE schedule was also effective in reducing proliferation of $\mathrm{H} 292$ and HCC827 cells, whereas an antagonistic effect was observed in the resistant cells, H460 and H1975. The underlying reasons for Gef $\rightarrow$ Gef + MTE acts different in gefitinib-resistant and -sensitive NSCLC cells deserve a further research. MTE also could enhance the tumor growth inhibitory effect of erlotinib, another common used TKI, by the three different combination procedures (see Additional file 1). In other studies, the exhibited synergistic or additive effects by different reagents combinations were also demonstrated. Paclitaxel followed by gefitinib was regarded as an effective treatment combination for NSCLC cell lines harboring EGFR mutations [36], while schedule-dependent synergistic effect was seen in the combinations of gefitinib and irinotecan in lung cancer cell lines [25].

Flow cytometric data revealed MTE and gefitinib alone or in combination caused different cell cycle arrest in H292 and HCC827 cells. They clearly induced G2/M arrest in $\mathrm{H} 292$ cells but caused S phase block in HCC827 cells, and this discrepancy may due to cell characteristics difference. The combination of $\mathrm{MTE} \rightarrow \mathrm{MTE}+$ gefitinib enhanced cell cycle arrest and apoptosis that induced by gefitinib in NSCLC cell lines and showed significant difference with each drug alone $(P<0.05)$. Taken together, these findings in part suggest that MTE $\rightarrow$ MTE + Gef could inhibit the growth of $\mathrm{H} 292$ and HCC 827 cells by inducing cell cycle arrest and apoptosis, and induction of apoptosis was seems to be more important in this study.

Over activation of ERK which is one of the main cell proliferation and survival factors. Studies showed that constitutive ERK1/2 activity in NSCLC cells promotes cellular survival and chemotherapeutic resistance [37]. In our research, the phosphorylation of ERK was significantly suppressed by gefitinib. MTE also decreased ERK activation to some extent. The combination of MTE $\rightarrow$ $\mathrm{MTE}+$ Gef treatment enhanced the inhibition of gefitinib on H292 and HCC827 cell growth. This prominent suppression of cell proliferation was correlated with an enhanced inhibition on ERK1/2 activation by the combination of MTE $\rightarrow$ MTE + Gef.

The PI3K/Akt/mTOR is an important intracellular signaling pathway in cell apoptosis, and this deregulated cascade is reported to contribute to lung cancer development and maintenance [38]. Frequent Akt activation and mTOR phosphorylation were found in 51\% of NSCLC patients and in 74\% of NSCLC cell lines [39]. NSCLC cell lines responsive to EGFR TKIs with growth arrest or apoptosis showed a down-regulation of the PI3K/Akt/mTOR pathway [40]. In this study, 
gefitinib prominently inhibited the activation of PI3K/ Akt/mTOR. MTE alone also moderately decreased the phosphorylation of these signaling molecules at a relative low concentration used in the study $\left(\sim \mathrm{IC}_{15}, 8 \mathrm{mg} / \mathrm{ml}\right)$. Furthermore, MTE $\rightarrow$ MTE + Gef treatment acted synergistically in disruption of the PI3K/Akt/mTOR signaling cascade and induction of apoptosis in H292 and HCC827 cells.

c-Met and EGFR receptors are widely expressed in cancer cells, and they have redundant effects on cell cycle progression, apoptosis, motility and metastasis and are potential targets for combination therapy. There is a cross-talk between the c-Met and EGFR signaling pathways in lung cancer [29]. EGFR and c-Met kinase activity is required for EGF-induced c-Met phosphorylation. The present study showed treatment with gefitinib abolished tyrosine phosphorylation of c-Met and EGFR as it demonstrated in other research [29,41]. Of course, the combination of MTE and gefitinib further decreased c-Met and EGFR phosphorylation. To our surprise, MTE alone at a relative minimal cytotoxic concentration also decreased c-Met and EGFR phosphorylation very strongly in the present study, suggesting it could inhibit both of these two growth factor receptors. However, MTE alone only showed inhibition tendency in the resistant cells according to our previous experiment [20]. The same phenomenon was also observed in above mentioned PI3K/Akt/mTOR pathway and ERK1/2. It suggests that MTE acted differently in gefitinib-sensitive and -resistant NSCLC cells. But what factors resulted in the difference of MTE activity is an interesting issue and needs a further study.

\section{Conclusion}

In summary, we have found MTE and gefitinib acted synergistically or additively with different combinations to suppress gefitinib sensitive NSCLC cell growth over treatments with either agent alone. Meanwhile, the cell cycle arrest and apoptotic cells were greatly promoted by $\mathrm{MTE} \rightarrow \mathrm{MTE}+$ Gef treatment, the most powerful combination. The underlying mechanisms of this remarkable cell growth inhibition were mostly ascribed to the significant suppression of ERK1/2 and PI3K/Akt/mTOR. The blocked crosstalk of EGFR and c-Met was also contributed to the prominent anti-tumor effect of $\mathrm{MTE} \rightarrow \mathrm{MTE}+\mathrm{Gef}$ treatment. However, there was no obvious difference between the effect of this combination in EGFR mutant cell line HCC827 and wild type cell line H292. Thus, our results suggest that MTE is a promising herbal medicine to improve gefitinib efficacy in NSCLC regardless of EGFR status. The different effect of MTE in gefitinib-sensitive and -resistant NSCLC cells needs further research.

\section{Additional file}

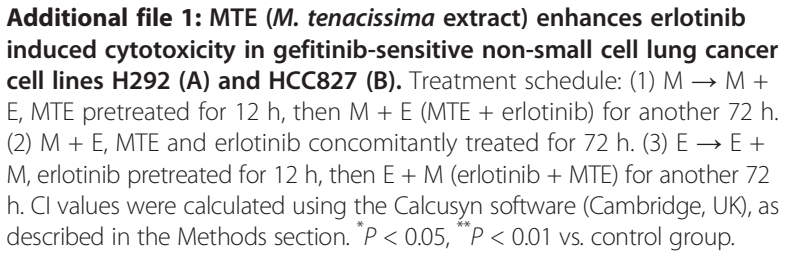

\section{Abbreviations}

MTE: Marsdenia tenacissima extract; EGFR: Epidermal growth factor receptor NSCLC: Non-small cell lung cancer; Cl: Combination index;

ECL: Chemiluminescence; EGF: Epidermal growth factor; TKI: Tyrosine kinase inhibitor.

\section{Competing interests}

The authors declare that they have no competing interest in the study.

\section{Authors' contributions}

SYH performed experiments, participated in experimental design and drafted the manuscript. HRD, WZ and FT carried out the experimental procedures and analyzed the data. PPL conceived of the study, participated in its design and coordination, and helped to draft the manuscript. All authors read and approved the final manuscript.

\section{Acknowledgements}

This research was supported in part by grants from Beijing Municipal Natural Science Foundation (No. 7112027), China Postdoctoral Science Foundation (No. 20090450009), and Beijing Municipal Traditional Chinese Medicine Technology Foundation (JJ2010-17).

\section{Author details}

${ }^{1}$ Key Laboratory of Carcinogenesis and Translational Research (Ministry of Education), Department of Integration of Chinese and Western Medicine, Peking University Cancer Hospital \& Institute, No. 52 Fucheng Road, 100142 Haidian District, Beijing, P.R. China. ${ }^{2}$ Central Laboratory of Biochemistry and Molecular Biology, Haidian District, P.R. China. ${ }^{3}$ Department of Cell Biology, Peking University Cancer Hospital \& Institute, 100142 Haidian District, Beijing, P.R. China.

Received: 19 November 2013 Accepted: 13 May 2014 Published: 22 May 2014

\section{References}

1. Hansen $\mathrm{HH}$ : Treatment of advanced non-small cell lung cancer. Br Med J 2002, 325:452-453

2. Mendelsohn J: Targeting the epidermal growth factor receptor for cancer therapy. J Clin Oncol 2002, 20:1s-13s.

3. Habib AA, Chun SJ, Neel BG, Vartanian T: Increased expression of epidermal growth factor receptor induces sequestration of extracellular signal-related kinases and selective attenuation of specific epidermal growth factor-mediated signal transduction pathways. Mol Cancer Res 2003, 1:219-233.

4. Veale D, Kerr N, Gibson GJ, Harris AL: Characterization of epidermal growth factor receptor in primary human non-small cell lung cancer. Cancer Res 1989, 49:1313-1317.

5. Laskin JJ, Sandler AB: Epidermal growth factor receptor: a promising target in solid tumours. Cancer Treat Rev 2004, 30:1-17.

6. Mendelsohn J, Baselga J: Epidermal growth factor receptor targeting in cancer. Semin Oncol 2006, 33:369-385.

7. Hynes NE, Lane HA: ERBB receptors and cancer: the complexity of targeted inhibitors. Nat Rev Cancer 2005, 5:341-354.

8. Gridelli C, De Marinis F, Di Maio M, Cortinovis D, Cappuzzo F, Mok T: Gefitinib as first-line treatment for patients with advanced non-small-cell lung cancer with activating epidermal growth factor receptor mutation: review of the evidence. Lung Cancer 2011, 71:249-257.

9. Sequist LV, Martins RG, Spigel D, Grunberg SM, Spira A, Jänne PA, Joshi VA, McCollum D, Evans TL, Muzikansky A, Kuhlmann GL, Han M, Goldberg JS, 
Settleman J, lafrate AJ, Engelman JA, Haber DA, Johnson BE, Lynch TJ: Firstline gefitinib in patients with advanced non-small-cell lung cancer harboring somatic EGFR mutations. J Clin Oncol 2008, 26:2442-2449.

10. Taron M, Ichinose Y, Rosell R, Mok T, Massuti B, Zamora L, Mate JL, Manegold C, Ono M, Queralt C, Jahan T, Sanchez JJ, Sanchez-Ronco M, Hsue V, Jablons D, Sanchez JM, Moran T: Activating mutations in the tyrosine kinase domain of the epidermal growth factor receptor are associated with improved survival in gefitinib-treated chemorefractory lung adenocarcinomas. Clin Cancer Res 2005, 11:5878-5885.

11. Takano T, Ohe Y, Sakamoto H, Tsuta K, Matsuno Y, Tateishi U, Yamamoto S, Nokihara H, Yamamoto N, Sekine I, Kunitoh H, Shibata T, Sakiyama T, Yoshida T, Tamura T: Epidermal growth factor receptor gene mutations and increased copy numbers predict gefitinib sensitivity in patients with recurrent non-small-cell lung cancer. J Clin Oncol 2005, 23:6829-6837.

12. Wang F, Wang S, Wang Z, Duan J, An T, Zhao J, Bai H, Wang J: Phosphorylated EGFR expression may predict outcome of EGFR-TKIs therapy for the advanced NSCLC patients with wild-type EGFR. J Exp Clin Cancer Res 2012, 31:65

13. Sequist LV, Bell DW, Lynch TJ, Haber DA: Molecular predictors of response to epidermal growth factor receptor antagonists in non-small-cell lung cancer. J Clin Oncol 2007, 25:587-595.

14. Mitsudomi T, Kosaka T, Endoh H, Horio Y, Hida T, Mori S, Hatooka S, Shinoda M, Takahashi T, Yatabe Y: Mutations of the epidermal growth factor receptor gene predict prolonged survival after gefitinib treatment in patients with non-small-cell lung cancer with postoperative recurrence. J Clin Oncol 2005, 23:2513-2520.

15. Ma C, Wei S, Song Y: T790M and acquired resistance of EGFR TKI: a literature review of clinical reports. J Thorac Dis 2011, 3:10-18.

16. Lee DH, Kim SW, Suh C, Yoon DH, Yi EJ, Lee JS: Phase II study of erlotinib as a salvage treatment for non-small-cell lung cancer patients after failure of gefitinib treatment. Ann Oncol 2008, 19:2039-2042.

17. Jiangsu Xinyi Xueyuan: Zhongyao Dacidian (Encyclopedia of Chinese Materia Medica). Shanghai: Shanghai Science and Technology Press; 1977:1976.

18. Zhang $H$, Tan AM, Zhang AY, Chen R, Yang SB, Huang X: Five new C21 steroidal glycosides from the stems of Marsdenia tenacissima. Steroids 2010, 75:176-183.

19. Luo SQ, Lin LZ, Cordell GA, Xue L, Johnson ME: Polyoxypregnanes from Marsdenia tenacissima. Phytochemistry 1993, 34:1615-1620.

20. Han SY, Zhao MB, Zhuang GB, Li PP: Marsdenia tenacissima extract restored gefitinib sensitivity in resistant non-small cell lung cancer cells. Lung Cancer 2012, 752:30-37.

21. Wang WY, Zhou Y, Zhang XJ, Gao TH, Luo ZF, Liu MY: A random study of xiao-ai-ping injection combined with chemotherapy on the treatment of advanced non-small cell lung cancer. Chin Clin Oncol 2009, 14:936-938.

22. Wang $K$, Guo ZX: Xiaoaiping injection combining with NP regiment in the treatment of 56 Patients with advanced lung cancer. J Basic Clin Oncol 2009, 22:47-48.

23. Huang ZQ, Tan H, Wang CY, Zhang HZ, Liu D, Zhou CZ, Liu X: Clinical research of combined xiaoaiping injection with chemotherapy on advanced non-small cell lung cancer. Chin Clinl Oncol 2007, 12:97-99.

24. Furugaki K, Iwai T, Shirane M, Kondoh K, Moriya Y, Mori K: Scheduledependent antitumor activity of the combination with erlotinib and docetaxel in human non-small cell lung cancer cells with EGFR mutation, KRAS mutation or both wild-type EGFR and KRAS. Oncol Rep 2010, 24:1141-1146

25. Shimoyama T, Koizumi F, Fukumoto H, Kiura K, Tanimoto M, Saijo N, Nishio K: Effects of different combinations of gefitinib and irinotecan in lung cancer cell lines expressing wild or deletional EGFR. Lung Cancer 2006, 53:13-21.

26. Chou TC: Drug combination studies and their synergy quantification using the Chou-Talalay method. Cancer Res 2010, 70:440-446.

27. Baselga J, Rischin D, Ranson M, Calvert H, Raymond E, Kieback DG, Kaye SB, Gianni L, Harris A, Bjork T, Averbuch SD, Feyereislova A, Swaisland H, Rojo F, Albanell J: Phase I safety, pharmacokinetic, and pharmacodynamic trial of ZD1839, a selective oral epidermal growth factor receptor tyrosine kinase inhibitor, in patients with five selected solid tumor types. J Clini Oncol 2002, 20:4292-4302.

28. Landi L, Minuti G, D'Incecco A, Cappuzzo F: Targeting c-MET in the battle against advanced nonsmall-cell lung cancer. Curr Opin Oncol 2013, 25:130-136.

29. Dulak AM, Gubish CT, Stabile LP, Henry C, Siegfried JM: HGF-independent potentiation of EGFR action by c-Met. Oncogene 2011, 30:3625-3635.
30. Guo A, Villén J, Kornhauser J, Lee KA, Stokes MP, Rikova K, Possemato A, Nardone J, Innocenti G, Wetzel R, Wang Y, MacNeill J, Mitchell J, Gygi SP Rush J, Polakiewicz RD, Comb MJ: Signaling networks assembled by oncogenic EGFR and c-Met. Proc Natl Acad Sci U S A 2008, 105:692-697.

31. Lemmon MA, Schlessinger J: Cell signaling by receptor tyrosine kinases. Cell 2010, 141:1117-1134.

32. Ono M, Hirata A, Kometani T, Miyagawa M, Ueda S, Kinoshita H, Fujii T, Kuwano M: Sensitivity to gefitinib (Iressa, ZD1839) in non-small cell lung cancer cell lines correlates with dependence on the epidermal growth factor (EGF) receptor/extracellular signal-regulated kinase 1/2 and EGF receptor/Akt pathway for proliferation. Mol Cancer Ther 2004, 3:465-472.

33. Zhao J: Nutraceuticals, nutritional therapy, phytonutrients, and phytotherapy for improvement of human health: a perspective on plant biotechnology application. Recent Pat Biotechnol 2007, 1:75-97.

34. Lee JY, Lee YM, Chang GC, Yu SL, Hsieh WY, Chen JJ, Chen HW, Yang PC: Curcumin induces EGFR degradation in lung adenocarcinoma and modulates p38 activation in intestine: the versatile adjuvant for gefitinib therapy. PLoS One 2011, 6:e23756.

35. Chang CM, Chang PY, Tu MG, Lu CC, Kuo SC, Amagaya S, Lee CY, Jao HY, Chen MY, Yang JS: Epigallocatechin gallate sensitizes CAL-27 human oral squamous cell carcinoma cells to the anti-metastatic effects of gefitinib (Iressa) via synergistic suppression of epidermal growth factor receptor and matrix metalloproteinase-2. Oncol Rep 2012, 28:1799-1807.

36. Cheng H, An SJ, Dong S, Zhang YF, Zhang XC, Chen ZH, Su J, Wu YL: Molecular mechanism of the schedule-dependent synergistic interaction in EGFR-mutant non-small cell lung cancer cell lines treated with paclitaxel and gefitinib. J Hematol Oncol 2011, 4:5

37. Brognard J, Dennis PA: Variable apoptotic response of NSCLC cells to inhibition of the MEK/ERK pathway by small molecules or dominant negative mutants. Cell Death Differ 2002, 9:893-904.

38. Méndez M, Custodio A, Provencio M: New molecular targeted therapies for advanced non-small-cell lung cancer. J Thorac Dis 2011, 3:30-56.

39. Balsara BR, Pei J, Mitsuuchi Y, Page R, Klein-Szanto A, Wang H, Unger M, Testa JR: Frequent activation of AKT in non-small cell lung carcinomas and preneoplastic bronchial lesions. Carcinogenesis 2004, 25:2053-2059.

40. Engelman JA, Jänne PA, Mermel C, Pearlberg J, Mukohara T, Fleet C, Cichowski K, Johnson BE, Cantley LC: ErbB-3 mediates phosphoinositide 3kinase activity in gefitinib-sensitive non-small cell lung cancer cell lines. Proc Natl Acad Sci U S A 2005, 102:3788-9373.

41. Puri N, Salgia R: Synergism of EGFR and c-Met pathways, cross-talk and inhibition, in non-small cell lung cancer. J Carcinog 2008, 7:9.

\section{doi:10.1186/1472-6882-14-165}

Cite this article as: Han et al:: Enhancement of gefitinib-induced growth inhibition by Marsdenia tenacissima extract in non-small cell lung cancer cells expressing wild or mutant EGFR. BMC Complementary and Alternative Medicine 2014 14:165.

\section{Submit your next manuscript to BioMed Central and take full advantage of:}

- Convenient online submission

- Thorough peer review

- No space constraints or color figure charges

- Immediate publication on acceptance

- Inclusion in PubMed, CAS, Scopus and Google Scholar

- Research which is freely available for redistribution 